\title{
Data Collection and Interpretation
}

\author{
Giuseppe Citerio ${ }^{1,2} \cdot$ Soojin $_{\text {Park }^{3}} \cdot$ J. Michael Schmidt ${ }^{3} \cdot$ Richard Moberg $^{4}$. \\ Jose I. Suarez ${ }^{5}$ Peter D. Le Roux ${ }^{6,7}$ • The Second Neurocritical Care Research \\ Conference Investigators
}

\begin{abstract}
Patient monitoring is routinely performed in all patients who receive neurocritical care. The combined use of monitors, including the neurologic examination, laboratory analysis, imaging studies, and physiological parameters, is common in a platform called multi-modality monitoring (MMM). However, the full potential of MMM is only beginning to be realized since for the most part, decision making historically has focused on individual aspects of physiology in a largely threshold-based manner. The use of MMM now is being facilitated by the evolution of bio-informatics in critical care including developing techniques to acquire, store, retrieve, and display integrated data and new analytic techniques for optimal clinical de-
\end{abstract}

The Second Neurocritical Care Research Conference Investigators are listed in "Appendix".

Peter D. Le Roux

jisuarez@bcm.tmc.edu; lerouxp@mlhs.org

1 Department of Health Science, University of Milan-Bicocca, and Neurointensive Care, Monza, Italy

2 Department of Emergency and Intensive Care, San Gerardo Hospital, Monza, Italy

3 Division of Neurocritical Care, Department of Neurology, Columbia University, New York, NY, USA

4 Moberg Research, Inc., Ambler, PA, USA

5 Division of Vascular Neurology and Neurocritical Care, Department of Neurology, Baylor College of Medicine, Houston, TX, USA

6 Lankenau Medical Center, Thomas Jefferson University, Philadelphia, PA, USA

7 Brain and Spine Center, Lankenau Medical Center, Suite 370, Medical Science Building, 100 East Lancaster Avenue, Wynnewood, PA 19096, USA cision making. In this review, we will discuss the crucial initial steps toward data and information management, which in this emerging era of data-intensive science is already shifting concepts of care for acute brain injury and has the potential to both reshape how we do research and enhance cost-effective clinical care.

Keywords Critical care - Data display - Data integration . Informatics $\cdot$ Neuromonitoring

\section{Introduction}

Prevention, detection, and management of secondary brain injury (SBI) are fundamental to patient care in the neurocritical care unit (NCCU). Consequently, neurocritical care depends in large part on careful and repeated assessment of clinical and laboratory findings, imaging studies, and bedside physiological data. This information comes in many forms e.g., written observation, ordinal data, images, pointin-time numeric laboratory values, or continuous data that also include waveforms. The NCCU therefore is a very data intense environment. However, the ability to record and interpret all this data often exceeds our ability to fully integrate it into patient care. Furthermore, treatment decisions generally are made in a reactive univariate fashion when a single threshold value is reached, e.g., treat a temperature $>38.5^{\circ} \mathrm{C}$ or an ICP $>20 \mathrm{mmHg}$, in large part because the human brain has difficulty judging the interaction between more than six variables at any when time. By contrast a physician may be faced with more than 200 variables when making management decisions about the average critical care patient. The interaction, rank order, trends over time, and relationship of these variables are only now beginning to be incorporated into patient care and given the complexity of 
the human brain and pathophysiology, likely are more important than a single variable alone.

An emerging concept in neurocritical care is use of multi-modality monitoring (MMM) defined as the simultaneous collection of data from multiple diverse sources associated with a single patient coupled with the ability to view the data in an integrated and time synchronized manner [1]. This concept is still in its infancy but rapidly evolving in parallel with advances in clinical informatics. Clinical- or bio-informatics deals with biomedical data, information, and knowledge including their acquisition, storage, retrieval, integration, display, analysis, and optimal use for clinical decision making. This is a dynamic and rapidly evolving field and with MMM is reshaping how we think about and care for patients in the NCCU. In this review, we will discuss the informatics infrastructure necessary for clinical MMM and for translational research. There are several interdependent components: (1) data acquisition, synchronization, integration, and storage, including data standardization of all relevant patient data into a single, searchable database, or data repository; (2) data display and visualization; (3) integrating multiple monitors and clinical information into clinical practice and workflow in a user-friendly and useful format; and (4) data processing, analysis, and interpretation to extract clinically relevant information from raw data and translate it into actionable clinical information [2].

\section{Data Acquisition and Standardization}

Data collection and archiving is the critical first step toward information management in the NCCU. In addition, to realize the full potential of data collected for clinical care and more importantly to drive research, it must be collected in a consistent manner at multiple sites around the world. At present, there are two basic approaches to acquire and store high-resolution data: (1) kiosk-type portable cart-based systems or (2) enterprise-level central (i.e., remote) servers [3]. Kiosk systems can be moved from room to room and tend to be easy to use by clinical staff for patient-specific clinical analyses, i.e., one patient at a time. However, data may not be stored in an open database format so limiting research efforts. With remote or central servers, all data from bedside monitors and devices are simultaneously collected and stored on a central server that has large computational and storage capacities. Such a system requires information technology support staff and security concerns can be a barrier to use. However, enterprise-level central servers facilitate research.

There are a variety of questions about data acquisition that still need to be answered. For example, what type of data should be collected and at what frequency? There are no specific guidelines for this but to be meaningful acquired data need to be comprehensive, i.e., all of the necessary data for a particular monitoring goal need to be collected and at a frequency higher than the duration of the events to be detected so as not to miss clinically significant events. Hence data collection at the highest possible sampling frequency is recommended but this depends on the application and its intended use. For some applications, data collected every $10 \mathrm{~min}$ are sufficient whereas for others, including many sophisticated computational algorithms, more frequent sampling may be required (e.g., every $5 \mathrm{~s}$, every second, every millisecond). The data, however, are meaningless unless also collected simultaneously, time synchronized, and displayed in an integrated fashion. Data also need to be annotated so that nurse, physicians, or researchers can search epochs of interest [4, 5]. How data are collected can have significant clinical and research implications. For example, much of our understanding about intracranial pressure (ICP) is derived from end-hour values recorded by a nurse. While manually recorded end-hour values have a good relationship with data recorded every $15 \mathrm{~min}$ [6] and reflect the computerized end-hour and mean hour values reasonably accurately, there can be significant differences between the manually recorded and continuous data [7]. Furthermore, between 20 and $40 \%$ of episodes (depending on their duration) of elevated ICP ( $>20 \mathrm{mmHg}$ ) may be missed when recorded manually and this error is more likely in unstable patients with a fluctuating ICP. This can have significant clinical and research implications [8] since the total dose of ICP [9] and even brief episodes of elevated ICP can adversely affect outcome [10].

Collection of continuous or near continuous data has a cost; for example, the necessity to review and edit the data so that artifact can be removed (data cleaning) and missing data dealt with e.g., device disconnection. In addition, the data from various sources need to be integrated and precisely time stamped. Once this is accomplished it then needs to be complemented by relevant clinical observations, laboratory results, and imaging data. To facilitate research, a modular approach may be envisaged with more detailed data collected according to the research (Fig. 1). This should include common terminologies, data models, and annotation standards so that the same tools are used and the same information collected across healthcare and research centers that can be based on the NINDS Common Data Elements (http://www.ninds.nih.gov/research/ clinical_research/toolkit/CDE_flyer_tbi.pdf). Several devices that can integrate ICU data are available now e.g., ICM+, ICU Pilot, Bedmaster XA, Axon Systems Eclipse Neurological Workstation, and the CNS Monitor. Collaborative projects such as BrainIt (Table 1) demonstrate that the recording of many physiological variables across 


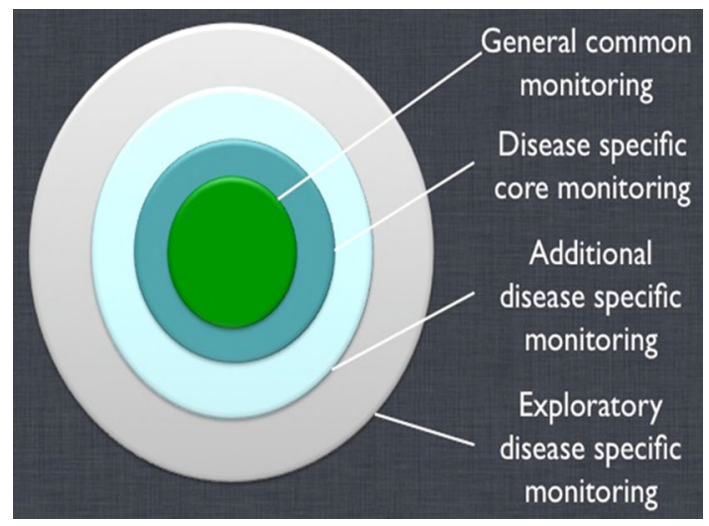

Fig. 1 Monitoring Common Data Elements. Taken from http://www. commondataelements.ninds.nih.gov/ProjReview.aspx\#tab=Introduction

multiple patients and across multiple centers is feasible and leads to new clinical insights and predictive tools [11-13].

\section{Data Display and Visualization}

The full potential of multimodal monitoring in the NCCU is under-realized but often this is not an issue of device design. A lot of sophisticated data are available but we tend to interpret information by looking at snapshots of individual aspects of physiology and react to it using thresholds. While meaningful integration of multiple variables at the bedside is beyond normal cognitive ability, some of this have to do with the time-pressured environment of critical care, an environment that is complex and can be cognitively disruptive and detract from direct care. Indeed, time motion studies show that very little time is actually spent in direct patient care [14, 15]. Physical and mundane reasons in the clinical environment, e.g., different monitors are in different locations and only some are visible from a central spot, contribute to this. In addition, patient care is driven by "interruptions" e.g., a nurse has two patients to look after or responses are driven by alarms [16]. There are also a lot of data that come in different forms e.g., text, ordinal, or image and from different sources e.g., a person, paper, or a computer that can make it a challenge to get information for one patient. Experienced clinicians often can sift through and disregard information to find meaning in data [17]. However, our ability to acquire data has outstripped our ability to understand it [18] which can contribute to "information overload" and preventable medical errors by providing too much data and not enough actionable information. A solution to this is efficient data display and visualization.

The goal of data visualization is to provide clinical decision support that improves situational awareness about the patient state. The way information is represented, however, can influence problem solving and what conclusions are drawn from the display [19]. In the NCCU, patient records usually are presented as text but a text display alone can increase the probability of error because users must collect, maintain, and integrate all the data mentally [20]. By contrast, graphical displays and integrated patient summaries that display all the information on a single screen can enhance speed and quality of clinical decision making [21]. Graphical displays also can improve recall and user satisfaction, whereas use of timeline displays helps find connections between events [22, 23].

There are several challenges to NCCU data display since multivariate patient data need to be presented in a single visual display. Furthermore, physiological data are dense because of its volume and high dimensionality. Combining too many elements in a single display can reduce critical event detection [23] and where there are multiple data streams computational algorithms can outperform clinicians [24]. Hence data visualization development needs to follow an iterative, human-centered design methodology to arrange information to support clinicians' the cognitive process but not aggravate clinical decision making [25]. Factors to consider in designing optimal data display and visualization are listed in Table 2. These factors can drive "decision support". For example, a bedside alarm (online analytic capability) is a "push" to the clinician. What is required, however, is a "pull" or early warning system i.e., "smart alarm." This may come in different forms and if effective can avoid alarm fatigue. First is a rule-based approach to data display and analysis that provides best practice and is based on hospital protocols and guidelines. The second approach is based on clinician surveys and provides expert opinion by combining a series of rules additively or multiplicatively depending on survey consensus. Finally there is an automatic (or unsupervised)

Table 1 BrainIT goals

To develop and disseminate standards for collection, analysis, and reporting of ICU monitoring and management data collected from brain injured patients

To develop and use a standardized database as a tool for hypothesis generation and development testing and validation of new data analysis methodologies

To provide an efficient multi-center infrastructure for generating evidence on the utility on new invasive and non-invasive intensive care monitoring and management methods 
Table 2 Factors to consider in optimal data display and visualization design

Integration of disparate types of data (e.g., devices and electronic medical records)

Storage of granular data in high fidelity and high resolution

Customizable graphical user interface that can be modified in an intuitive fashion

Accessible user interface to incentivize annotations

Ability to Refer to Time-Synced Annotations

Online analytic capability (locked for clinical safety)

Flexible middleware or a Universal platform

Guideline driven or Experimentally proven analyses

Connection to well-annotated representative database

Clinical context-aware decision support

approach that provides a data-driven approach using Baynesian networks to integrate patient-specific information.

\section{Connectivity and Integration}

The biggest challenge to translational research in neurocritical care complexity science is capturing granular data in a usable fashion from medical devices. In part, this is influenced by the data sources that vary greatly in type, e.g., baseline risk, periodic clinical data, laboratory findings and biomarkers, neuroimaging, and continuous data, and in frequency or regularity. In addition, device-associated factors including acquisition, time, and storage can affect data display. Often digital output from a device is in a proprietary or unique format and real-time data output is not a universal feature. However, interoperability is a marketable feature and standardization is likely to evolve.

Multi-modality monitoring (through clinical informatics) by definition requires integration of multiple monitors in a user-friendly format. There are three broad themes to be considered: (1) connectivity, (2) consistency by adopting standards so that everyone can seamlessly collect comprehensive patient data, and (3) creativity once there is connectivity and consistency. Creativity comes in many formats e.g., (1) an integrated clinical environment; (2) automated, exportable protocols, and guidelines to guide care; (3) the ICU as a learning environment (simulation); and (4) automating data collection in clinical trials.

\section{Connectivity}

The computer industry has figured this out, but there are still obstacles to connecting medical devices in the medical device industry. There are several reasons for this: (1) vital signs monitors use one manufacturer that tend to be geographic or institution based, (2) some monitors have data outputs, many don't or if they do they are many different forms, (3) some devices have no real-time data output (e.g., pumps), (4) there are incomplete data (e.g., no units), (5) incorrect use of communication standards, and (6) data are sent out into an IT system made up of multiple vendors among others. "Health IT and Patient Safety: Building Safer Systems for Better Care" published by the National Academies Press, in 2012 details the potential for errors in Health IT associated with the lack of safety standards and regulation. There is an evolving standard and regulatory system that remains a moving target that the FDA is looking at but to integrate multiple monitors several factors need to be considered (Fig. 2). The goal is that any data sent from a device get into the medical record (database) correctly. The first task is to develop interfaces between the various devices (plug and play). This will create multiple signals that require time synchronization and validation before storage and analysis (quality). Displays need to be integrated, comprehensive and bedside friendly, and allow for data review, event marking, provide point-of-care instruction and tutorials (embedded education). In turn, the integrated data should further connect to a database such that data from today's patients can help manage tomorrows patients (the Google approach). Several devices exist e.g., CNS Monitor ICU Pilot, ICM+ but currently each uses their own proprietary technology, different file formats, user interfaces, and types of data. Moving forward research in the NCCU will need to standardize connectivity. This will occur through collaboration between industry, federal agencies, and neurointensivists.

\section{Consistency}

To realize the full potential of data collected in the NCCU, standards need to be adopted so that everyone can seamlessly collect comprehensive patient data. The medical device industry has not yet fully adapted standards but this is evolving as MMM evolves. While most devices have data output ports (analog, serial, USB, and Ethernet) for data acquisition, there is no universally adopted standard that facilitates multimodal data acquisition and integration in a clinical setting; each one often uses its own communication protocol to transfer its data. Even simply linkingspecific device data with a specific patient can be difficult. In addition, there currently is no adopted standard for medical device time management and so time-synchronizing data from multiple devices are challenging because most devices maintain their own internal clocks and do not support updating clock time from the network. Without a "master clock" ensuring that all the values and waveforms acquired at the same time "line up" exactly in synch, interpreting the information and understanding the interrelationships is difficult, if not impossible. 


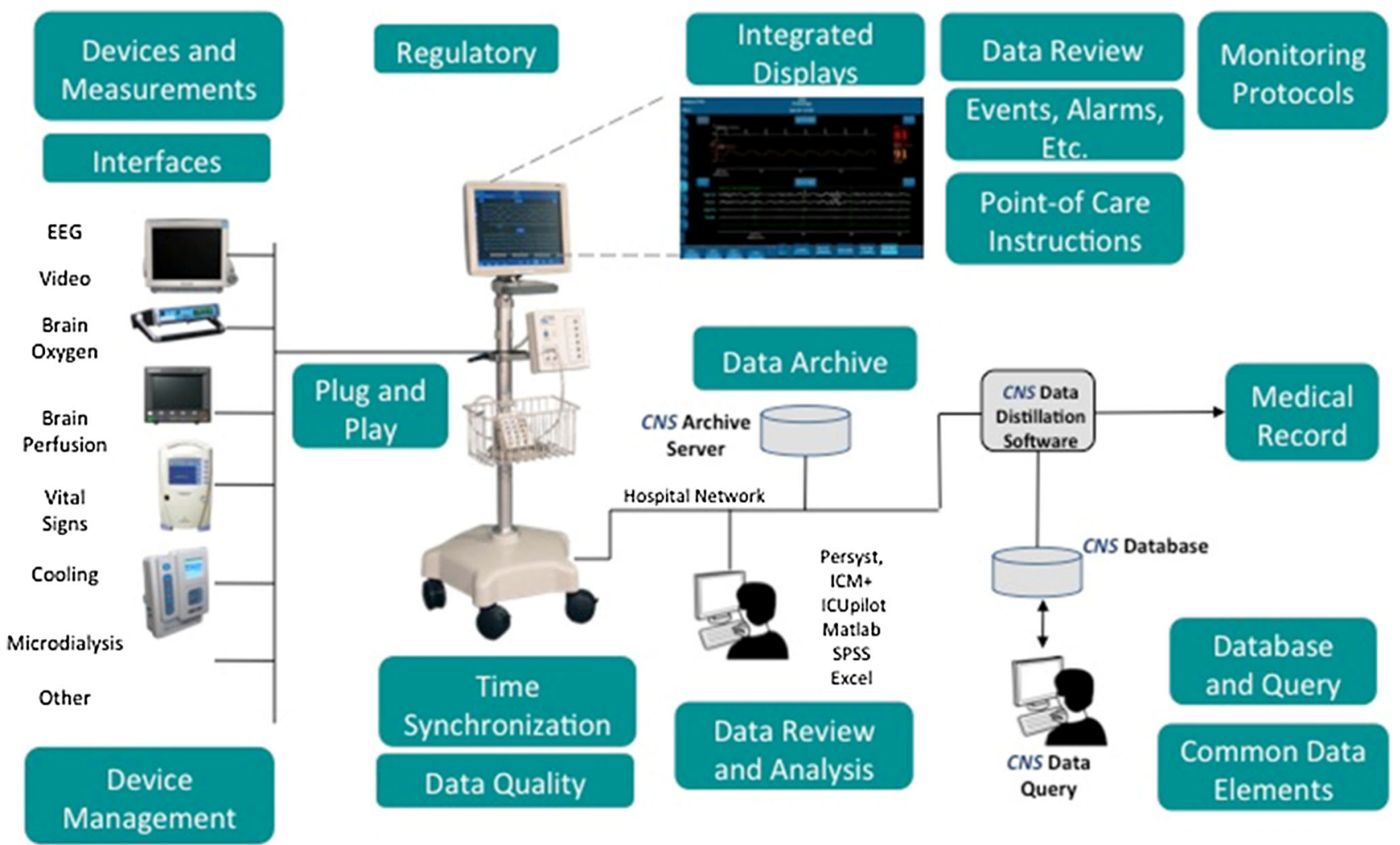

Fig. 2 Integrating multiple medical monitors: some of the things that need to be considered

There are a variety of needed standards: (1) medical device communications, (2) naming of data and devices, (3) time, (4) data characteristics e.g., quality and granularity, and (5) plug-in protocol standards among others. ISO/IEEE 11073 is a standard for medical device data communications. There also are names for medical devices including the Universal Medical Device Nomenclature System ${ }^{\mathrm{TM}}$ (UMDNS) through the ECRI Institute (https://www.ecri.org) and Unique Device Identifiers (UDI) through the FDA. These various standards can be adopted but to move MMM and clinical informatics forward requires device manufacturers to utilize data communication standards and allow time synchronization of their devices. Standards also are required on how much data are collected. This depends on the intended use, i.e., the frequency of data needs to be consistent with the application. For research, data mining, and hypothesis generation, every effort should be made to collect and store annotated data in a data warehouse, preferably using a standard open format, for translational research. In the NCCU, high-frequency data acquisition may be necessary for more precise evaluation of SBI $[4,8]$.

\section{Creativity}

Connectivity and consistency, i.e., once we have consistent data, are the key to unlocking creative uses of multimodal patient data. In the ICU of the future, the patient will be at the center of a vast computer system such that the patient interfaces with ICU and hospital networks. Three factors are needed. First is the association of all data sources and their output with the ICU patient. Second is time synchronization across all bedside devices to achieve a stable electronic flow sheet and medical record. Third is "interoperability" among data sources, middleware, and the medical record. This process aligns the proprietary medical device data output with industry standards (www.ihe.net), i.e., the middleware can recognize the data.

ICU care and research can be advanced through several creative clinical informatics approaches and use of ICU middleware. For example, multiparametric alarms or alarms that capture alerts can convert them into actionable information by transmitting them to specific personnel. Similarly, intelligent alarm systems or data sniffers that monitor ICU data and the EMR can identify patients at risk and create personalized alarms. In research, data collection in clinical trials can be automated or protocols can be specifically adapted to the patient and the environment. Clinical guidelines also can be adapted to the patient: when the patient's context is known a computer-driven guideline manager can personalize the guideline. Furthermore, "resource links" can compensate for variations in the training of personnel. Hence "generic" protocols can be exported 
into specific hospital or research sites since the local integrated informatics environment adapts the generic protocol to the specific site or patient.

\section{Data Analysis and Interpretation}

The NCCU is a very data-intensive environment but paradoxically despite all the data there is often no information. There are several reasons for this. In particular, physicians often need to integrate information in their heads and few analytical tools are available at the bedside. For example, many monitors display trends based on 3-5 s time-averaged parametric data from the monitor (often next to the waveforms) and for the most part analysis has been limited to classic linear statistical methods (mean, median, range, standard deviation). Even these have been difficult to perform at the bedside in real time. In addition, it is difficult for data to be indexed, searched, and assembled to provide accurate information to treat patients, because the original context of the data is lost.

The promise of clinical informatics lies in the potential to use a wide array of advanced and novel analytical techniques on high-resolution, multidimensional, multimodal physiological data to develop a better understanding of the complex relationships among various physiological parameters and so enhance the ability to predict future events and improve outcome. The aviation industry has achieved improved safety and efficiency by enhancing a pilot's situational awareness, i.e., taking all the available information from the multitude of dials and instruments in the cockpit to improve the pilots understanding of a airplane's situation relative to its environment, a simple actionable item. In the NCCU what is required is a systems engineering approach with advanced analytical tools to transform raw data into actionable information. Standard biostatistical methods are not sufficient to elucidate the important information we need in part because physiological relationships are not linear. Physiology also has high dimensionality. The dimension of a space or object is informally defined as the minimum number of coordinates needed to specify any point within it. As the number of dimensions increases, the number of possible dimensions increases factorially. For example, if examining a plot of CPP versus $\mathrm{PbtO}_{2}$, there are many other metrics that influence this e.g., MAP and ICP, FiO2, cardiac output, and $\mathrm{Hgb}$ to name a few. Processing also is limited conceptually since clinicians in the ICU may be confronted with 200 related variables, yet human beings can only determine the relationship between two variables unassisted, i.e., we think in a univariate fashion in a multivariate world. Conceptually, analysis has been restricted to linear and univariate statistical models. Alarm limits are based on arbitrary thresholds (often extremes of physiologic systems) without regard for dynamic interactions between variables. Consequently, order sets have thresholds, so in the absence of any understanding of what is happening we are relegated to very simple interventions based on only the most extreme limits of an organ system.

So where do we go? Simple indices may be calculated using informatics to add information to patient assessment. For example, calculating the area under the curve (AUC) above a specific cut point e.g., temperature $>38.5^{\circ} \mathrm{C}$ or ICP $>20 \mathrm{mmHg}$ provides a more robust measure of pathophysiologic "dose" that then can be used to track therapy. Another conceptually simple method is use of correlation coefficients between parameters. For example, the moving correlation coefficient between MAP and ICP is used to generate the pressure reactivity index (PRx). This provides information about cerebral autoregulation and may be used to identify patient-specific (or optimal) CPP and ICP thresholds that in turn demonstrate a more robust relationship with outcome than generic population-based thresholds [26, 27].

Techniques for nonlinear analysis (complex systems analysis) can also be borrowed from other disciplines e.g., genomics, mathematics, engineering, and complexity science. There are a variety of techniques including moving correlation coefficients, Dynamic Bayesian networks, artificial neural networks, Kohonen self-organizing maps, hierarchical cluster analysis, time series analysis (variability, approximate entropy, and detrended fluctuation analysis among others). Data infrastructures should be adopted that allow a wide range of analysis methods to be applied to data. For example, hierarchical cluster analysis can identify different clusters composed of different relationship structures among the data that are not discernable to the clinician and more accurately predict outcomes and patient trajectory $[28,29]$. Conceptualizing patients then as existing in physiologic and pathophysiologic "states" has led to the idea that therapies may need to be redirected toward facilitating transitions toward favorable physiologic states rather than "fixing" particular physiologic variables $[30,31]$.

Time series analysis that measures variation over time also has been applied to clinical research. For example, this has been applied to heart rate analysis by evaluating intervals between consecutive QRS complexes. Decreased heart rate variability is associated with poor outcome in cardiac disease and is thought to reflect pathology and a reduced ability to respond to perturbations [32]. Similarly, reduced ICP variability may be a better outcome predictor than other measures of ICP e.g., mean [33]. Approximate entropy (ApEn) provides a measure of the degree of randomness within a series of data. Less critically ill patients have higher variance and ApEn (more variable, less 
periodic signals), whereas loss of variability and lower ApEn is linked with parallel deterioration of organ dysfunction and high mortality [34]. Similarly, ICP ApEn decreases as elevated ICP develops [35]. Detrended fluctuation analysis describes "fractal" scaling behavior of variability in physiological signals (similar patterns of variation across multiple time scales). In neurocritical care, altered "fractal scaling" of the ICP signal is associated with poor outcome [36].

Looking ahead there is no universal equation for complex systems. Instead research and clinical care in the NCCU require high-resolution data acquisition and a suite of tools for physiologic signal processing and analysis, using both classical techniques and novel methods based on statistical physics and nonlinear dynamics. Complex nonlinear systems are present in biology and so the application of complexity science (chaos theory, nonlinear dynamics, complex adaptive systems, systems theory) that deals with the behavior and properties of complex nonlinear systems likely will provide new insights into normal physiologic relationships and the pathobiology of critical illness [36-39]. This is important since the clinical course and outcome of individual patients in the NCCU depend on a large and variable number of components (physiologic variables) that display marked variability over time, a high degree of connectivity and interdependence and sensitive dependence to initial conditions (small initial differences may cause dramatic effects in long-term dynamics-the "butterfly effect"). Currently, we simplify our care to a single numeric threshold, whereas information about variability may be more important than information of a single value, or trend of values. Furthermore, information about interactions between systems may be more important than variability within a system and Information about the emergent order of the entire system may be even more important than individual interactions. Using new analytic tools in the NCCU therefore should provide a more accurate model of physiology and new insights into therapy.

Acknowledgments The Second Neurocritical Care Research Conference was funded by the National Institutes of Health (NINDS) (R13 NS077610, PI: Suarez JI), the Integra Foundation, and the Baylor St Luke's Medical Center in Houston, TX.

\section{Appendix}

List of Second Neurocritical Care Research Conference Investigators in alphabetical order Abdelhak Tamer, MD, Henry Ford Hospital, Detroit, MI; Aisiku Imolgele, MD, UT Houston, Houston, TX; Alderazai Yazan, MD, UT Houston, Houston, TX; Safdar Ansari, MD, Baylor College of Medicine, Houston, TX; Armstead William, MD,
University of Pennsylvania, Philadelphia, PA; Badjatia Neeraj, MD, Columbia University, New York, NY; Bele Sylvia, MD, Regensburg, Germany; Bernard Francis, MD, University of Montreal, Montreal, Quebec; Bershad Eric, MD, Baylor College of Medicine, Houston, TX; Bowman Deanna, Hemedex Inc, Cambridge, MA; Bowman Frederick, PhD, Hemedex Inc, Cambridge, MA; Calvillo Eusebia, RN, Baylor College of Medicine, Houston, TX; CastilloAbrego Guadalupe, MD, Panama City, Panama; Cava Luis, MD, University of Colorado, Denver, CO; Chang Cherylee, MD, Queens Medical Center, Honolulu, HI; Chesnut Randall, MD, University of Washington, Seattle, WA; Chhangani Sanjeev, MD, Massachusetts General Hospital, Boston, MA; Chumbe Maria, MD, Insituto Neurologico, Lima, Peru; Ciro-Quintero Juan Diego, MD, Clinica Las Americas, Medellin, Colombia; Citerio Giuseppe, MD, San Gerardo Hospital, Monza, Italy; Clark Robert, MD, PhD, University of Pittsburgh, Pittsburgh, PA, Coffey Lisa, Hemedex Inc, Cambridge, MA; Corry Jesse, MD, Marshfield Clinic, Marshfield, WI; Coplin William, MD, Wayne State University, Detroit, MI; Dhar Rajat, MD, Washington University, St Louis, MO; Diringer Michael, MD, Washington University, St Louis, MO; Divani Afshin, PhD, University of Minnesota, Minneapolis, MN; Domingues Joao, MD, Brazil; Elzammar Ziad, MD, SUNY Upstate Medical University, Syracuse, NY; Ezzedine Mustapha, MD, University of Minnesota, Minneapolis, MN; Figolt Doug, Codman Neuro, Raynham, MA; Figueroa Stephen, MD, UT Southwestern, Dallas, TX; Freeman William, MD, Mayo Clinic, Jacksonville, Fl; Fugate Jennifer, MD, Mayo Clinic, Rochester, MN; Fuse Akira, MD, Nippon Medical School, Tokyo, Japan; Geocadin Romergryko, MD, Johns Hopkins University, Baltimore, MD; Giraldo Elias, MD, Drexel University College of Medicine, Philadelphia, PA; Girardeau Rosemarie, RN, Integra Neurosciences, Plainsboro, NJ; Glassner Stuart, MD, University of Miami, Miami, Fl; Gomez Mauricio, MD, Los Angeles, CA; Goodman Jerry, MD, Baylor College of Medicine, Houston, TX; Gopinath Shankar, MD, Baylor College of Medicine, Houston, TX; Gress Daryl, MD, University of Viriginia, Charlottesville, VA; Grocott Hilary, MD, University of Manitoba, Winnipeg, MB; Gupta Puneet, MD, UT Southwestern, Dallas, TX; Haider Huma, Baylor College of Medicine, Houston, TX; Halinger Mangat, MD, Cornell University, New York, NY; Hall Casey, MD, Emory University, Atlanta, GA; Hall Christiana, MD, UT Southwestern, Dallas, TX; Hannawi Yousef, MD, Baylor College of Medicine, Houston, TX; Hemphill J Claude, MD, PhD, UCSF, San Francisco, CA; Hernandez Olga H, MD, Instituto Neurologico de Colombia, Medellin, Colombia; Hinson Holly, MD, OHSU, Portland, OR; Hocker Sara, MD, Mayo Clinic, Rochester, MN; Jones Elizabeth, RN, Baylor St Luke's Medical 
Center, Houston, TX; James Michael, MD, Duke University, Durham, NC; Jichici Draga, MD, McMaster University, Hamilton, ON; Kapinos Gregory, MD, North Shore Hospital LIJ, Long Island, NY; Kirkpatrick Peter, University of Cambridge, Cambridge, UK; Ko Nerissa, MD, UCSF, San Francisco, CA; Kofke Andrew, MD, University of Pennsylvania, Philadelphia, PA; Koterba Edwin, MD, University of Sao Paulo, Brazil; Kramer Andreas, MD, Calgary, AB; Kuramatsu Joji, MD, Univ of Erlangen, Germany; Kutsogiannis Demetrios, MD, University of Alberta, Edmonton, AB; Latorre Julius, MD, SUNY Upstate Medical University, Syracuse, NY; Lazaridis Christos, MD, Baylor College of Medicine, Houston, TX; Ledoux David, MD, North Shore Hospital LIJ, Long Island, NY; Lee Jennifer, MD, Baylor College of Medicine, Houston, TX; Leroux Peter, MD, University of Pennsylvania, Philadelphia, PA; Liebschner Michael, $\mathrm{PhD}$, Baylor College of Medicine; Livesay Sarah, RN, PhD, Baylor St Luke's Medical Center, Houston, TX; Lopez Hubiel, MD, CEDIMAT, Dominican Republic; Luedtke Michael, Codman Neuro, Raynham, MA; Maas Andrew, MD, University Hospitals Antwerp, Belgium; Mandava Pitchaiah, MD, $\mathrm{PhD}$, Baylor College of Medicine, Houston, TX; Manno Edward, MD, CCF, Cleveland, OH; March Karen, RN, Integra Neurosciences, Plainsboro, NJ; Martin Renee, $\mathrm{PhD}$, MUSC, Charleston, SC; McCullough Laurence, PhD, Baylor College of Medicine, Houston, TX; Mejia Jorge H, MD, Fundacion Valle del Lili, Colombia; Miller Emmy, $\mathrm{RN}, \mathrm{PhD}$, Baylor College of Medicine, Houston, TX; Mizrahi Eli, MD, Baylor College of Medicine, Houston, TX; Moberg Richard, PhD, Moberg Research, Ambler, PA; Molyneaux Bradley, MD, Massachusetts General Hospital, Boston, MA; Muehlschlegel Susanne, MD, Dr med, UMass, Worcester, MA; Murthy Harish, MD, Good Samaritan Hospital, San Jose, CA; Murthy Santosh, MD, Baylor College of Medicine, Houston, TX; Norris Gregory, MD, Wayne State University, Detroit, MI; Nguyen Thuy, PharmD, Baylor St Luke's Medical Center, Houston, TX; Nyquist Paul, MD, PhD, Johns Hopkins University, Baltimore, MD; Olson Daiwai, RN, PhD, Duke University, Durham, NC; O'Phelan Kristine, MD, University of Miami, Miami, FL; Palesch Yuko, PhD, MUSC, Charleston, SC; Parikh Gunjan, MD, Columbia University, New York, NY; Park Soojin, MD, University of Pennsylvania, Philadelphia, PA; Prado Darwin, MD, Rio de Janeiro, Brazil; Rajajee Venkatakrishna, MD, University of Michigan, Ann Arbor, MI; Ramirez Jorge, MD, San Jose, Costa Rica; Venkatasubba Rao Chethan P, MD, Baylor College of Medicine, Houston, TX; Rehman Mohammed, MD, Henry Ford Hospital, Detroit, MI; Rethnam Rajesh, MD, Houston, TX; Rigamonti Andrea, MD, University of Toronto, Toronto, ON; Robertson Claudia, MD, Baylor College of Medicine, Houston, TX; Rosand Jonathan, MD,
Massachusetts General Hospital, Boston, MA; Rymer Marilyn, MD, St Luke's Hospital, Kansas City, MO; Sachdev Harmeet, MD, Good Samaritan Hospital, San Jose, CA; Sarwal Aarti, MD, Wake Forest University, Winston Salem, NC; Schmidt Michael J, PhD, Columbia University, New York, NY; Schneck J MICHAEL, MD, Loyola University, Chicago, Il; Schneider Hauke, MD, Technical University, Dresden,Germany; Seder David, MD, Tufts University, Boston, MA; Shah Shreyansh, MD, Baylor College of Medicine, Houston, TX; Sheth Kevin, MD, University of Maryland, Baltimore, MD; Shutter Lori, MD, University of Cincinnati, Cincinnati, OH; Siegel Sherry, MD, Integra Neurosciences, Plainsboro, NJ; Silva Ricardo, MD, Rio de Janeiro, Brazil; Smirnakis Stelios M, MD, PhD, Baylor College of Medicine, Houston, TX; Souter Michael, MD, University of Washington, Seattle, WA; Staykov Dimitre, MD, University of Erlangen, Germany; Stiver Shirley, MD, UCSF, San Francisco, CA; Suarez Jose I, MD, Baylor College of Medicine, Houston, TX; Sung Gene, MD, University of Southern California, Los Angeles, CA; Suri Muhammad Fareed, MD, University of Minnesota, Minneapolis, MN; Tirschwell David, MD, University of Washington, Seattle, WA; Torbey Michel, MD, OSU, Columbus, OH; Trapuzzano Gary, Moberg Research, Ambler, PA; Treggiari Miriam, MD, University of Washington, Seattle, WA; Turgeon Alexis, MD, Universite Laval, Quebec, Quebec; Urfy Zain, MD, Baylor College of Medicine, Houston, TX; Varelas Panayiotis, MD, PhD, Henry Ford Hospital, Detroit, MI; Villalobos Luis, MD, Buenos Aires, Argentina; Wijman Christine, MD, PhD, Stanford University, Palo Alto, CA; Yonas Howard, MD, University of New Mexico, Albuquerque, NM; Yuste Fernando, MD, BARD Medical, Covington, GA; Zafar Sahar, MD, Baylor College of Medicine, Houston, TX; Zygun David, MD, University of Calgary, Calgary, AB.

\section{References}

1. Le Roux P, Menon DK, Citerio G, Vespa P, Bader MK, Brophy GM, Diringer MN, Stocchetti N, Videtta W, Armonda R, Badjatia N, Böesel J, Chesnut R, Chou S, Claassen J, Czosnyka M, De Georgia M, Figaji A, Fugate J, Helbok R, Horowitz D, Hutchinson P, Kumar M, McNett M, Miller C, Naidech A, Oddo M, Olson D, O'Phelan K, Provencio JJ, Puppo C, Riker R, Robertson C, Schmidt M, Taccone F, Consensus Summary Statement of the International Multidisciplinary Consensus Conference on Multimodality Monitoring in Neurocritical Care: a statement for healthcare professionals from the Neurocritical Care Society and the European Society of Intensive Care Medicine. Neurocrit Care. 2014 Sep 11. [Epub ahead of print].

2. Buchman TG. Novel representation of physiologic states during critical illness and recovery. Crit Care. 2010;14:127.

3. Martich G, Waldmann C, Imhoff M. Clinical informatics in critical care. J Intens Care Med. 2004;19:154. 
4. Hemphill JC, Andrews P, De Georgia M. Medscape. Multimodal monitoring and neurocritical care bioinformatics. Nat Rev Neurol. 2011;7(8):451-60.

5. Signorini DF, Piper IR, Jones PA, Howells TP. Importance of textual data in multimodality monitoring. Crit Care Med. 1997;25:2048-50.

6. Venkatesh B, Garrett P, Fraenkel DJ, Purdie D. Indices to quantify changes in intracranial and cerebral perfusion pressure by assessing agreement between hourly and semi-continuous recordings. Intens Care Med. 2004;30:510-3.

7. Zanier ER, Ortolano F, Ghisoni L, Colombo A, Losappio S, Stocchetti N. Intracranial pressure monitoring in intensive care: clinical advantages of a computerized system over manual recording. Crit Care. 2007;11(1):7.

8. Hemphill JC 3rd, Barton CW, Morabito D, Manley GT. Influence of data resolution and interpolation method on assessment of secondary brain insults in neurocritical care. Physiol Meas. 2005;26(4):373-86.

9. Vik A, Nag T, Fredriksli OA, Skandsen T, Moen KG, SchirmerMikalsen K, Manley GT. Relationship of "dose" of intracranial hypertension to outcome in severe traumatic brain injury. J Neurosurg. 2008;109(4):678-84.

10. Stein DM, Hu PF, Brenner M, Sheth KN, Liu KH, Xiong W, Aarabi B, Scalea TM. Brief episodes of intracranial hypertension and cerebral hypoperfusion are associated with poor functional outcome after severe traumatic brain injury. J Trauma. 2011;71(2):364-74.

11. Piper I, Citerio C, Chambers I. The BrainIT Group: concept and core dataset definition. Acta Neurochir. 2003;145:615-29.

12. Piper I, Chambers I, Citerio G, et al. The brain monitoring with Information Technology (BrainIT) collaborative network: EC feasibility study results and future direction. Acta Neurochir (Wien). 2010;152:1859-71.

13. Stell A, Sinnott R, Jiang J, Donald R, Chambers I, Citerio G, Enblad P, Gregson B, Howells T, Kiening K, Nilsson P, Ragauskas A, Sahuquillo J, Piper I. Federating distributed clinical data for the prediction of adverse hypotensive events. Philos Trans A. 1898;2009(367):2679-90.

14. Chisholm CD, Weaver CS, Whenmouth L, Giles B. A task analysis of emergency physician activities in academic and community settings. Ann Emerg Med. 2011;58(2):117-22.

15. Tang Z, Weavind L, Mazabob J, Thomas EJ, Chu-Weininger MY, Johnson TR. Workflow in intensive care unit remote monitoring: a time-and-motion study. Crit Care Med. 2007;35(9): 2057-63.

16. Malhotra S, Jordan D, Shortliffe E, Patel VL. Workflow modeling in critical care: piecing together your own puzzle. J Biomed Inform. 2007;40(2):81-92.

17. Woods DD, Patterson ES, Roth EM. Can we ever escape from data overload? A cognitive systems diagnosis. Cogn Technol Work. 2002;4:22-36.

18. De Turck F, Decruyenaere J, Thysebaert P, et al. Design of a flexible platform for execution of medical decision support agents in the intensive care unit. Comput Biol Med. 2007;37:97-112.

19. Zhang J, Norman DA. Representations in distributed cognitive tasks. Cogn Sci. 1994;18:87-122.

20. Woods D. The cognitive engineering of problem representations. Human-computer interaction and complex systems, vol. 169. New York: Academic Press; 1991. p. 188.

21. Koch S, Staggers N, Weir C, Agutter J, Liu D, Westenskow D. Integrated information displays for ICU nurses: field observations, display design, and display evaluation. Beverly Hills: Sage; 2010. p. 932-6.
22. Alonso D, Rose A, Plaisant C, Norman K. Viewing personal history records: a comparison of tabular format and graphical presentation using LifeLines. Behav Inf Technol. 1997;17:24962.

23. Effken JA, Loeb RG, Kang Y, Lin ZC. Clinical information displays to improve ICU outcomes. Int J Med Inform. 2008;77: 765-77.

24. M.I.T. Laboratory of Computational Physiology, Physionet challenge. http://physionet.org/challenge/2009/. 2009.

25. Faiola A, Newlon C. Advancing critical care in the ICU: a human-centered biomedical data visualization systems. Ergon Health Asp Work Comput. 2011;2011:119-28.

26. Lazaridis C, DeSantis SM, Smielewski P, Menon DK, Hutchinson P, Pickard JD, Czosnyka M. Patient-specific thresholds of intracranial pressure in severe traumatic brain injury. J Neurosurg. 2014;120(4):893-900.

27. Aries MJ, Czosnyka M, Budohoski KP, Steiner LA, Lavinio A, Kolias AG, Hutchinson PJ, Brady KM, Menon DK, Pickard JD, Smielewski P. Continuous determination of optimal cerebral perfusion pressure in traumatic brain injury. Crit Care Med. 2012;40(8):2456-63.

28. Sorani MD, Hemphill JC 3rd, Morabito D, et al. New approaches to physiological informatics in neurocritical care. Neurocrit Care. 2007;7:45-52.

29. Cohen MJ, Grossman AD, Morabito D, Knudson MM, Butte AJ, Manley GT. Identification of complex metabolic states in critically injured patients using bioinformatic cluster analysis. Crit Care. 2010;14:R10.

30. Rixen D, Siegel J, Friedman H. Sepsis/SIRS, physiologic classification, severity stratification, relation to cytokine elaboration and outcome prediction in posttrauma critical illness. J Trauma Injury Infect Crit Care. 1996;41(4):581-98.

31. Buchman TG. Physiologic stability and physiologic state. J Trauma. 1996;41:599-605.

32. Kleiger RE, Miller JP, Bigger JT Jr, Moss AJ. Decreased heart rate variability and its association with increased mortality after acute myocardial infarction. Am J Cardiol. 1987;59:256-62.

33. Kirkness CJ, Burr RL, Mitchell PH. Intracranial pressure variability and long-term outcome following traumatic brain injury. Acta Neurochir. 2008;102:105-8.

34. Papaioannou VE, Maglaveras N, Houvarda I, Antoniadou E, Vretzakis G. Investigation of altered heart rate variability, nonlinear properties of heart rate signals, and organ dysfunction longitudinally over time in intensive care unit patients. J Crit Care. 2006;21:95-103.

35. Hornero R, Aboy M, Abasolo D, McNames J, Goldstein B. Interpretation of approximate entropy: analysis of intracranial pressure approximate entropy during acute intracranial hypertension. IEEE Trans Biomed Eng. 2005;52:1671-80.

36. Burr RL, Kirkness CJ, Mitchell PH. Detrended fluctuation analysis of intracranial pressure predicts outcome following traumatic brain injury. IEEE Trans Biomed Eng. 2008;55: 2509-18.

37. Buchman TG. Nonlinear dynamics, complex systems, and the pathobiology of critical illness. Curr Opin Crit Care. 2004;10:378-82.

38. Goldberger AL, Filley GF. Complex systems. Proc Am Thorac Soc. 2006;3(6):467-71

39. Peelen L, de Keizer NF, Jonge Ed, Bosman RJ, Abu-Hanna A, Peek N. Using hierarchical dynamic Bayesian networks to investigate dynamics of organ failure in patients in the Intensive Care Unit. J Biomed Inform. 2010;43:273-86. 